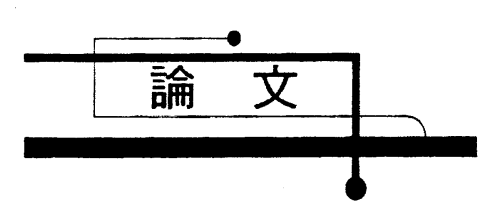

\title{
Axial and Circumferential Film Flow Rate Prediction in a Horizontal Annular Two-Phase Flow Using the FIDAS-3DT Code*
}

\author{
曹治 平 ${ }^{* *}$ 菅原悟** \\ TSO Chih-Ping SUGAWARA Satoru
}

\begin{abstract}
Prediction of developing and transient characteristics of circumferential and axial film velocities in a horizontal annular two-phase flow is carried out with the FIDAS-3DT computer code developed in Japan that can provide three-field representation in subchannel flow using the full conservative equations and simple constitutive relations. The specifications are those from a reported air-water experiment in a $3.9 \mathrm{~m}$ by $32 \mathrm{~mm}$ diameter pipe. Steady, fully developed axial flow rates seem to be over-predicted compared to the experimental results. A corrective procedure is suggested which takes into account the radial variation in the axial velocity profile.
\end{abstract}

Keywords : Annular flow, Horizontal flow, Three-field model, Film flow rates, Subchannel flow code, Entrainment, Deposition.

\section{INTRODUCTION}

The modelling of horizontal annular two-phase flow has been more problematic than vertical flow due to the effect of the gravitational force breaking the axial symmetry, resulting in a thicker liquid film at the pipe bottom. Early theoretical analyses were based on condensation models such as those proposed by Chaddock (1957) and Chato (1962), where the upper film was modelled only by circumferential flow of the condensate. Butterworth's mass balance model (1972, 1973) attempted to account for circumferential and axial flow in the whole pipe by considering the effects of entrainment and deposition. Better entrainment modelling was proposed by Hutchinson et al.(1974) and James \& Burns (1979), while Wilkes et al. (1980) improved

* Received 11 April 1989

** School of Mechanical \& Production Engineering, Nanyang Technological Institute, Singapore.

*** Reactor Engineering Section, O-arai Engineering Center, Power Reactor and Nuclear Fuel Development Corporation, O-arai, Ibaraki, Japan. 
on the deposition modelling. Laurinat et al.(1985) considered a formulation that also included a stress balance on the film, which gave rise to a recirculating secondary flow. This model was also tested by Lin et al.(1985), and reported to give better results. James et al. (1987) proposed further refinement by theorizing on a variable deposition flux. Recently, Fukano \& Ousaka (1989) formulated a model where the disturbance waves play a central role in the transfer of liquid in the circumferential direction.

The present study obtains the characteristics of an adiabatic flow along a horizontal pipe by applying the full mass, momentum and energy macroscopic conservation equations for incompressible flow to the three fluids, namely, water film, water droplets and water vapour, using the FIDAS-3DT Code (1987). This new approach to horizontal flow has the advantage of predicting not just the steady-state results, but also the transient and developing results along the length of the pipe. The equations are solved by the finite difference method over divided cells in the channel, and the analysis is carried out to simulate the experiment conditions in the work of Butterworth and Pulling (1973). In an earlier paper (Tso and Sugawara, 1990), results for liquid water film volume fraction variations around the circumference, throughout the channel, and as a function of time, were presented. The fully developed steady state calculated results for the water film were found to compare fairly well with the experimental results. The present paper discusses results for the axial and circumferential velocities and water film flow rates. Limited results for steady state flow only had been reported in Tso and Sugawara (1989).

\section{ANALYSIS}

The FIDAS-3DT Code has been proven to be useful for vertical subchannel analysis, especially in predicting dryout and post-dryout in a channel or in rod bundles (Sugawara et al., 1989 ; Sugawara and Miyamoto, 1990).

The code and the experimental conditions simulated for this problem have been described in Tso and Sugawara (1990). Basically the Code provides three-field representation of two-phase flow in a horizontal pipe by formulating twelve basic field equations consisting of three continuity equations, three energy equations and six momentum equations. The twelve field equations together with the volume fraction conservation relation enable solution for the following thirteen parameters; the six velocities, the three volume fractions, the three specific enthalpies, and the pressure. As the full set of governing equations and further details have been prsented in the earlier paper, only the set for the water film is listed here, in Appendix $\mathrm{A}$ as equations (A1) to (A4). In the present study, the fluids are water and air, the three fields being the continuous water film around the pipe wall, the continuous air and the entrained water droplets suspended in the air in the central core, as shown in Fig. 1 .

The conservation equations need to be supported by the pertinent hydrodynamic constitutive relations for closure of solution, and generally these relations are still 


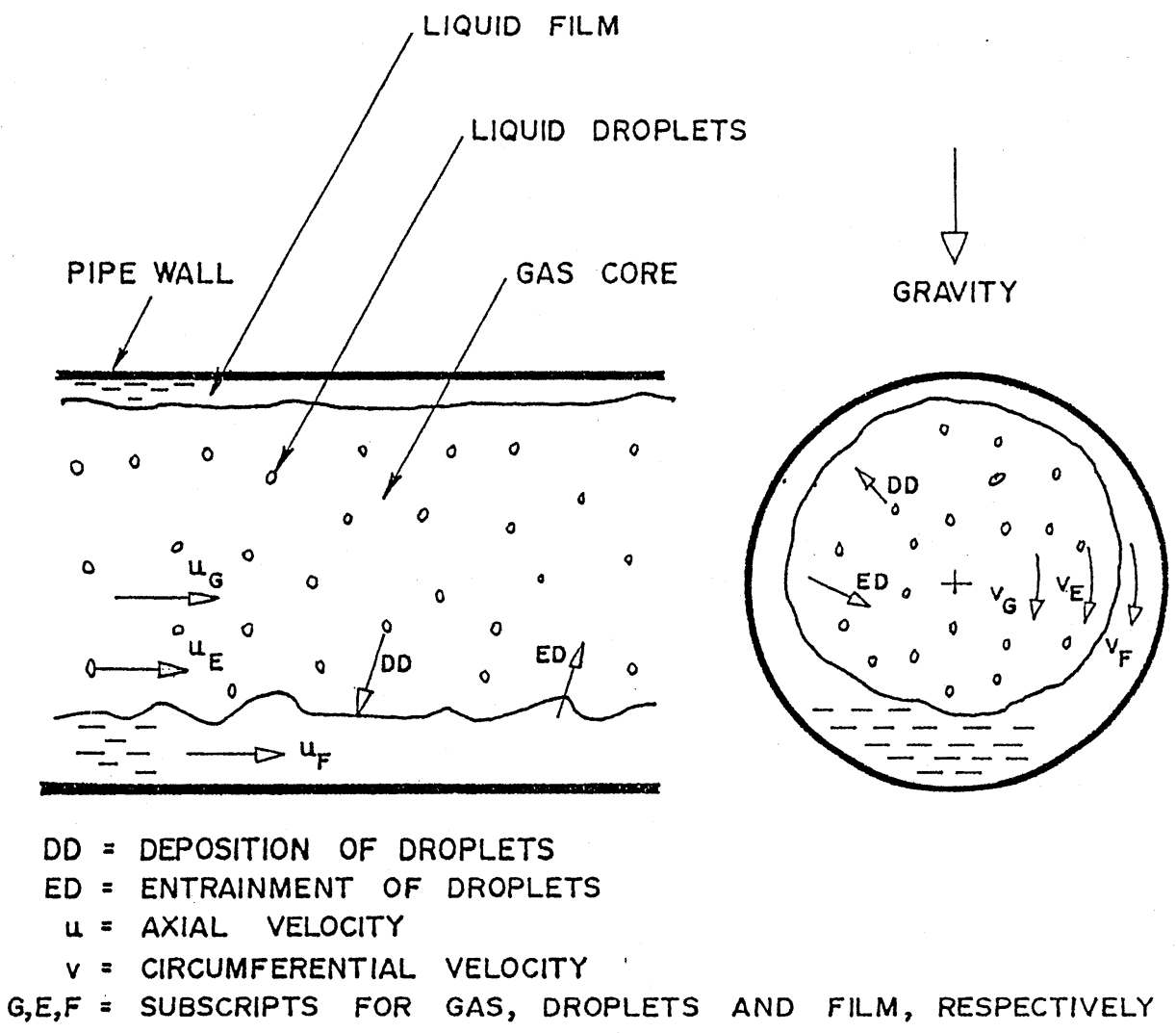

Fig. 1 Three-fluid model in horizontal annular flow.

not well understood in multi-phase flow. In this study, simple expressions will be used, as provided by the Code, with no major distinction being made between horizontal and vertical flow apart from the difference in the gravitational force terms in the equations.

Firstly consider droplet entrainment and deposition rates. A number of experiments and correlations had been reported and they were reviewed by Hewitt and Hall Taylor (1970), Ueda (1981), Ishii (1982) and others. Specific modelling for application to horizontal flow was also mentioned in the Introduction. For droplet entrainment rate, Huchinson and Whalley (1972) correlated their data as a function of a dimensionless group given by the product of the interfacial shear stress and the film thickness divided by the surface tension, based on the force balance at the wavy interface between liquid film and vapor. Ueda (1981) also presented a correlation similar to that of Huchinson and Whalley, and Ueda's correlation is used in the present Code. Kataoka and Ishii (1982) proposed a more complicated correlation on the basis of entrainment inception criteria and force balance at the wavy interface.

The deposition of droplets is usually expressed by a deposition coefficient. Paleev and Filippovich (1966) derived a correlation from his experimental data in atmospheric 
air-water flow, and which gave the deposition coefficient as a function of gas velocity, gas Reynolds number, and a ratio of droplet density to gas density. Whalley (1977) recommended a coefficient in terms of the surface tension, while Bennett et al. (1966) and Saito (1978) correlated the coefficient as a funciton of droplet concentration. Recently, Sugawara (1988) proposed a model based on the turbulent diffusivity of entrained droplets in the the vapor core. In the present study, the simple model by Farmer et al.(1970), as described in Appendix B is used.

\begin{tabular}{|l|l|}
\hline POSITION & PARAMETER \\
\hline X-NODE & $\alpha_{F}, \alpha_{E}, \alpha_{G}$ \\
$h_{F}, h_{E}, h_{G}$ \\
$P$
\end{tabular}
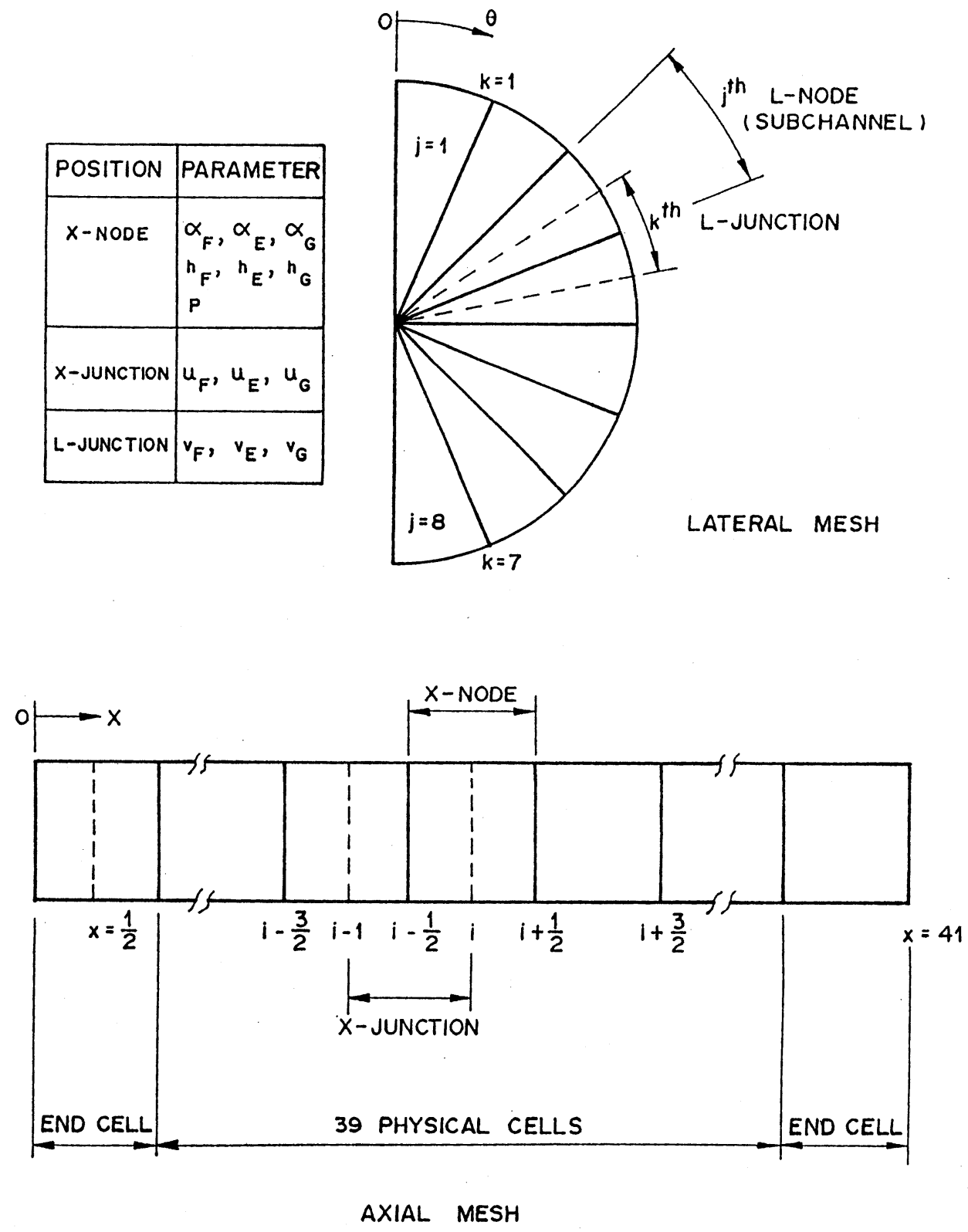

Fig. 2 Mesh structure. 
Turning now to the interfacial resistances, the wall-liquid drag force is based on the Blasius form as in Appendix B. The vapor-liquid drag force is based on the model by Ueda (1981). Other models in the literature includes the wave velocity model and the Wallis model (1969). For the vapor-droplet drag force the conventional single phase correlation is used, while the average droplet size is determined from the critical Weber number as detailed in Appendix B. Finally, in the turbulent momentum and mass diffusion modelling, the generalized Prandtl mixing length theory is used.

The top of Fig. 2 shows the division of the pipe section into 16 lateral sectors representing 16 subchannels, where, by virtue of symmetry about the vertical line only 8 subchannels are considered. The subchannels or $L$-nodes are numbered $j=1$ to 8 , with their respective angular positions indicated by $\theta$, as measured from the pipe top. The circumferential velocities are specified between two adjacent subchannels or at the "L-junctions". They are denoted by $v_{F}, v_{E}$ and $v_{G}$ where the subscripts $F, E$ and $G$ refer to the water film, the entrained water droplets and the air respectively.

Turning now to the axial mesh shown at the bottom of Fig. 1 , the pipe is divided into 39 physical cells with two extra cells, one at each end. These " $x$-nodes" are the locations specifying the properties volume fractions $\alpha_{F}, \alpha_{E}, \alpha_{G}$ enthalpies $h_{F}, h_{E}, h_{G}$ and pressure $p$. Between adjacent $\mathrm{x}$-nodes are defined the "x-junctions" which specify the axial velocities $u_{F}, u_{E}, u_{G}$.

The conditions simulated in the present study are those in a horizontal pipe with a test section of length $3.9 \mathrm{~m}$ and internal diameter $32 \mathrm{~mm}$. Two sets of entrance conditions are separately simulated. The first is for a "high water flow" at a water flow rate of $0.128 \mathrm{~kg} / \mathrm{s}$ and an air flow rate of $0.025 \mathrm{~kg} / \mathrm{s}$. The second set is for a "low water flow" at a water flow rate of $0.064 \mathrm{~kg} / \mathrm{s}$ and an air flow rate of $0.051 \mathrm{~kg} / \mathrm{s}$. According to the two-phase flow pattern map by Scott (1963), both sets of flow conditions are expected to exhibit dispersed annular flow characteristics. The specifications for the inlet conditions for both sets are the inlet velocities and the inlet volume fractions of water, air and droplets; enthalpies and densities of water and air being at $300 \mathrm{~K}$. The initial condition at the pipe outlet is the exit pressure of 2.5 bar. All heat transfer mechanisms are neglected.

The inlet velocities and volume fractions are obtained based on the above inlet flow rates of water, air and droplets, the droplet flowrate being zero, as well as on the average experimental film thickness taken to be distributed uniformly at the inlet. It is noteworthy that the outlet film thickness distribution in the present study is obtained by using the time marching method. That is, steady state is obtained as an asymptotic solution of the transient simulation under constant boundary conditions. Parametric study on the outlet film thickness distribution have shown that the predicted values are not sensitive to the initial condition of the film thickness distribution at the inlet, since the test section used in the experiment of Butterworth and Pulling is long enough to obtain the equilibrium condition at the outlet. 


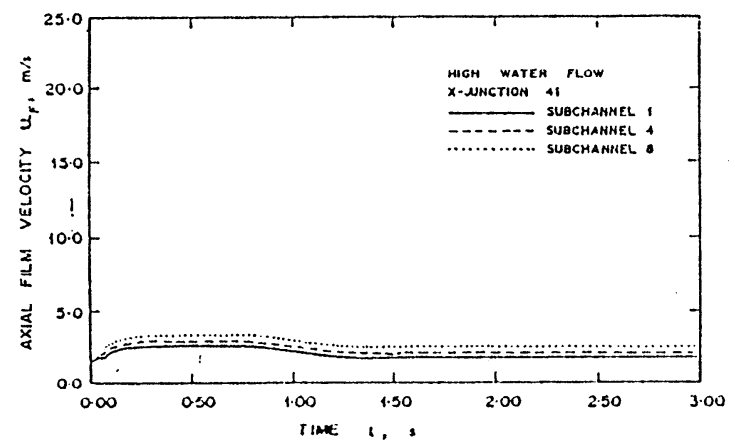

(a)

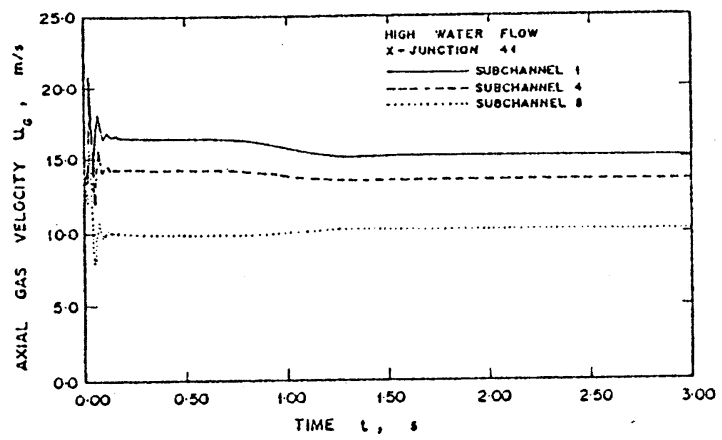

(b)

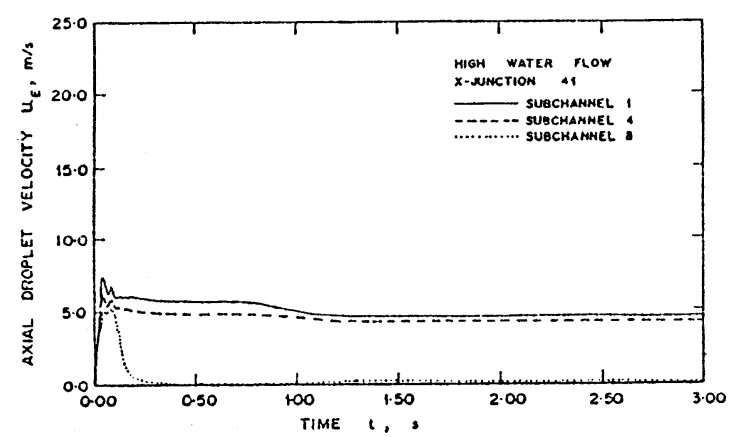

(c)

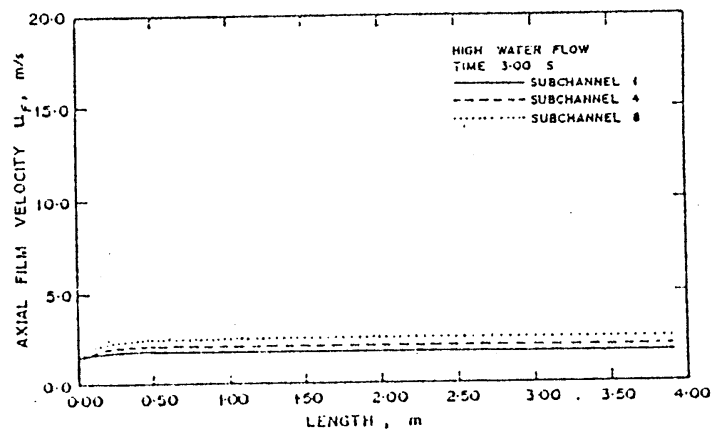

(d)

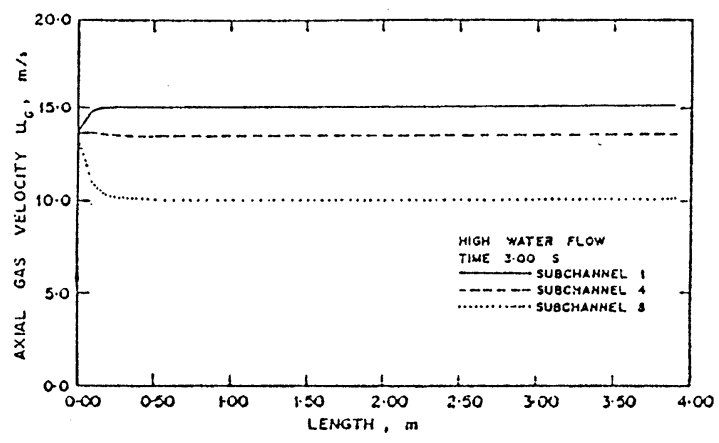

(e)

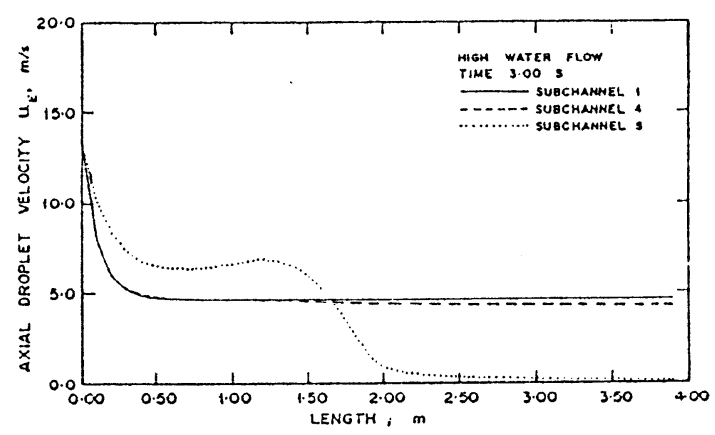

(f)

Fig. 3 Results for axial velocities. 


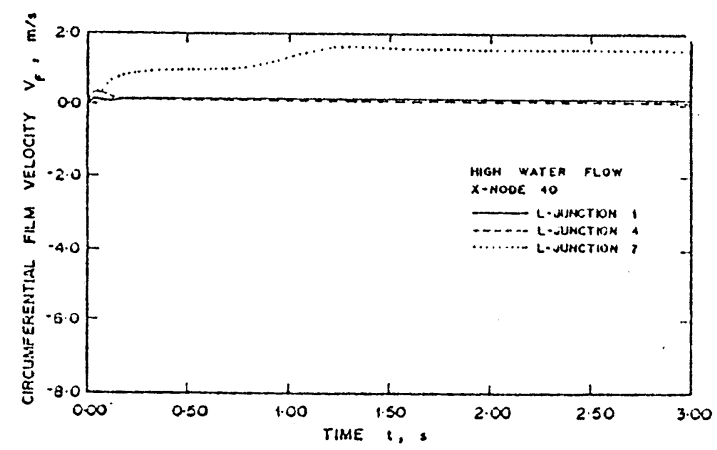

(a)

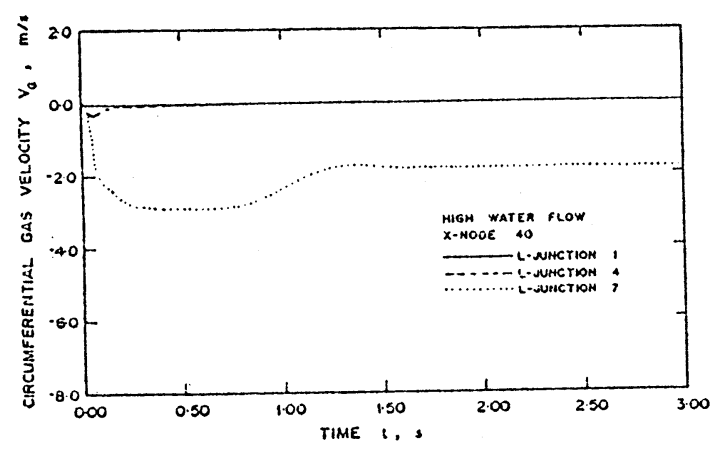

(b)

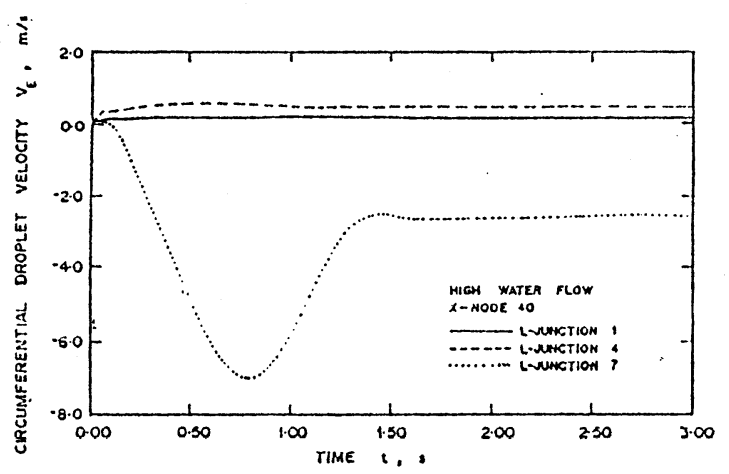

(c)

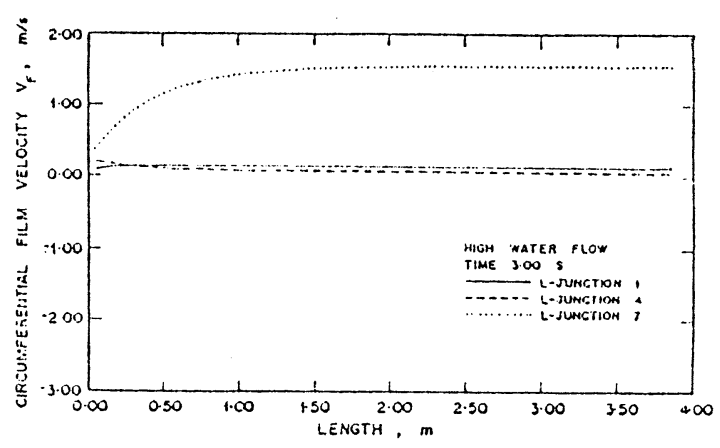

(d)

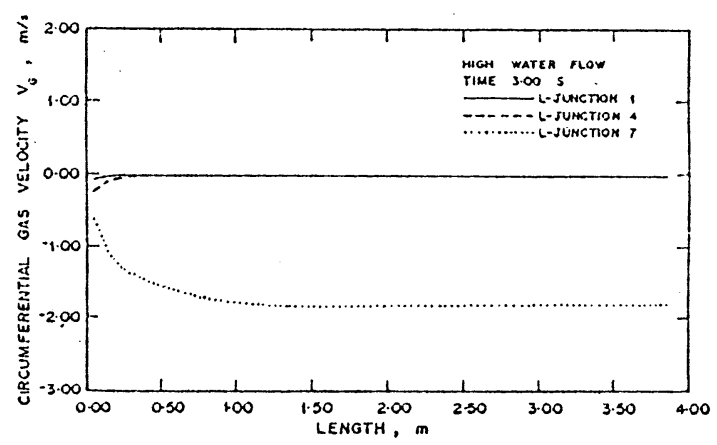

(e)

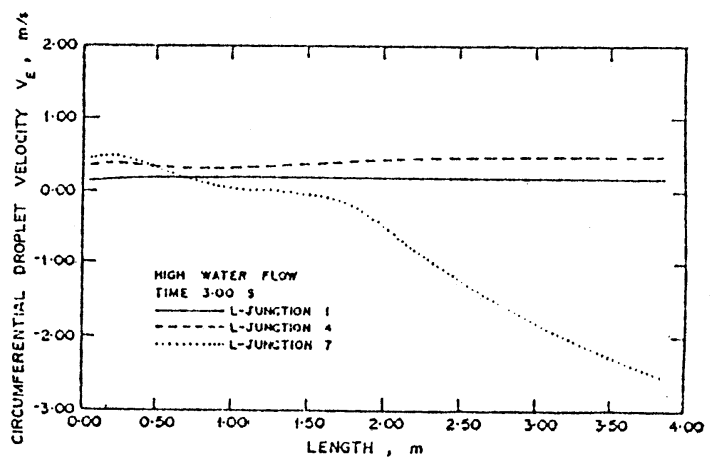

(f)

Fig. 4 Results for circumferential velocities. 


\section{3 . RESULTS}

The finite difference form of the conservative and constitutive equations are applied to the mesh cells, and the resulting equations solved numerically on the Fujitsu FACOM-M-380 computer. Results for the velocity distributions are given for the high water flow case only. Figure 3 shows the results for axial velocities $u_{F}, u_{G}$ and $u_{E}$, with the time history graphs for 3 subchannels being displayed in (a), (b) and (c) for up to $3.00 \mathrm{~s}$ at the pipe exit. It is seen that conditions may be regarded as steady beyond 1.5 to $2.0 \mathrm{~s}$ for all 3 fluids, and the axial film velocity increases from the top of the pipe (subchannel 1 ) to the bottom (subchannel 8 ). The reverse is true for the gas and droplet velocity, where the highest velocities are at the top. In (d), (e) and (f), are shown the axial variation graphs, where axial velocities for the same 3 subchannels 1,4 and 8 are plotted along the length of

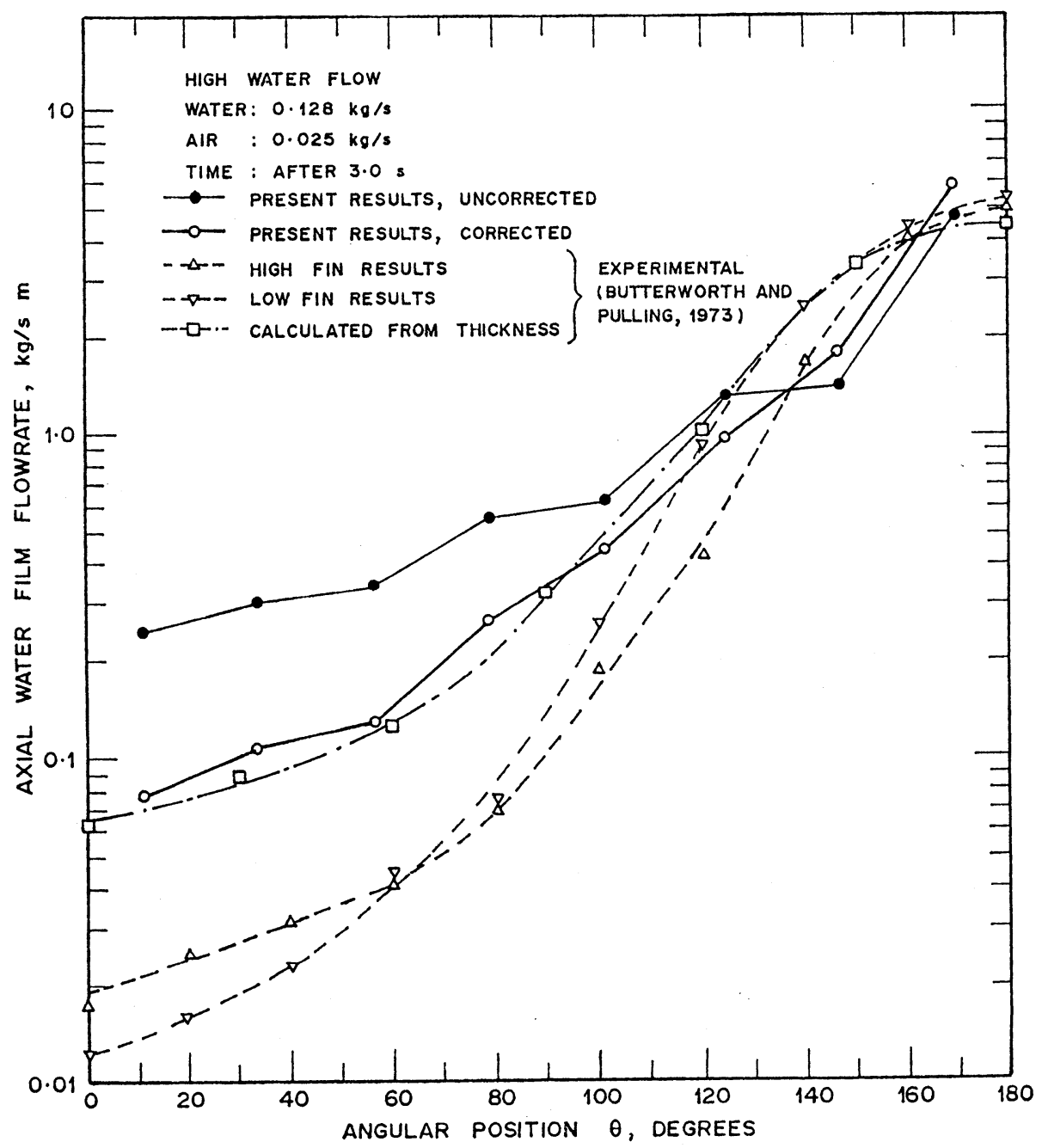

Fig. 5 Angular distribution for axial flow rates in high water flow. 
the pipe, at the final physical time of $3.00 \mathrm{~s}$. It is clear that the velocities have reached the fully developed conditions at the pipe exit.

Turning now to the circumferential velocity distribution results for L-junctions 1, 4 and 7 in Fig. 4, the graphs in (a), (b) and (c) again show that steady state is achieved already at a physical time of $3 \mathrm{~s}$. The circumferential velocities are lower at the pipe top than at the bottom, though the directions of $v_{F}$ and $v_{G}$ seem to be anti-clockwise for $L$-junction 7 . With respect to (d), (e) and (f) of Fig. 4 , fully-developed status are achieved by $v_{F}$ and $v_{G}$, but not by $v_{E}$ in L-junction 7 . However, this may be of no great significance to the prediction of the water film flow rate, since the volume fraction of droplets is extremely small.

The results for velocities may be converted into volumetric flow rates simply by

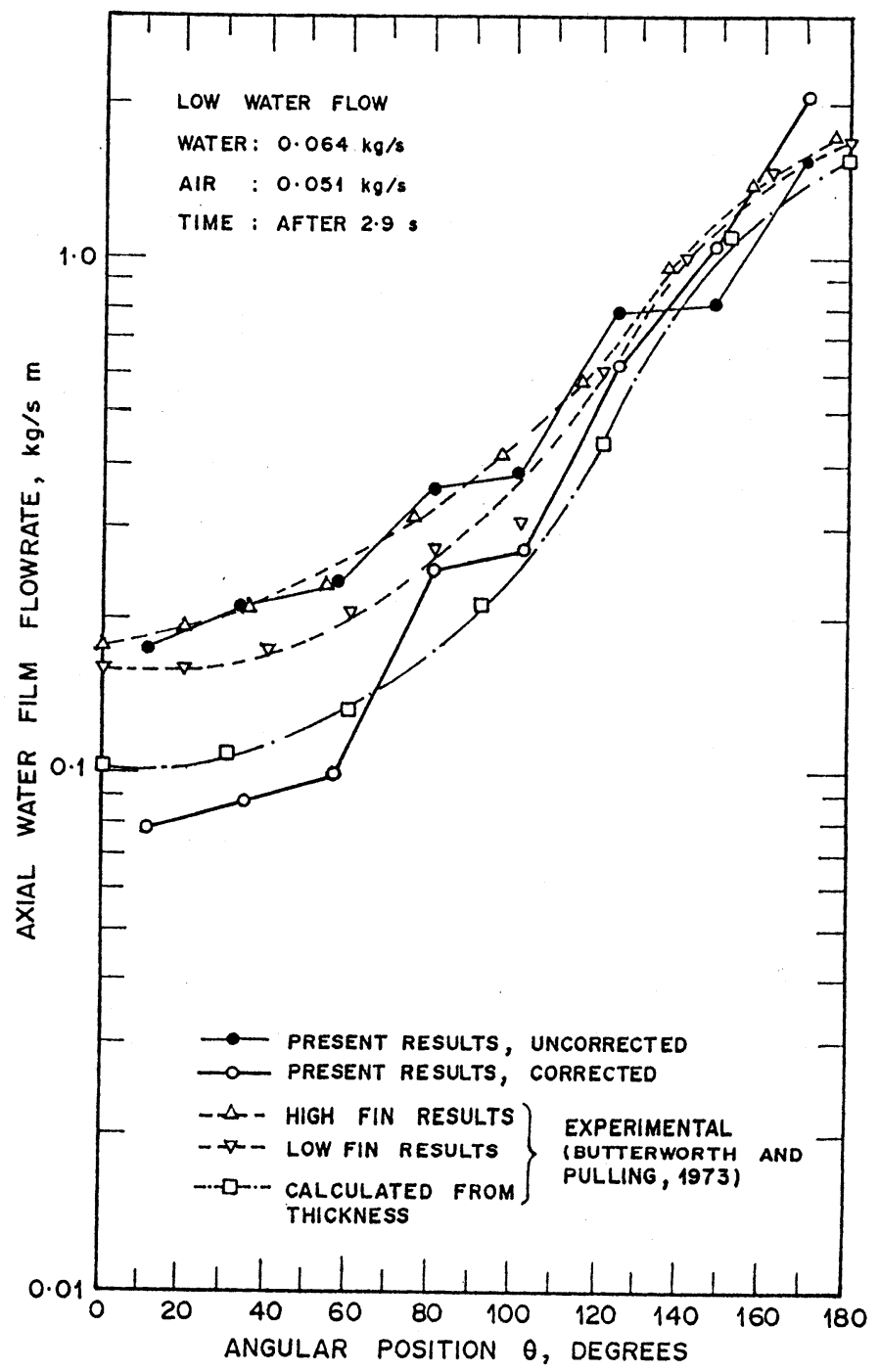

Fig. 6 Angular distribution for axial flow rates in low water flow. 
multiplying with the product of the appropriate volume fraction and the appropriate area. Results for the volume fractions for the higher water flow case were presented in Tso and Sugawara (1990). Figure 5 shows the final results for the fully developed, steady angular distribution of axial film flow rates in high water flow, where the angular position is measured clockwise from the pipe top as indicated in Fig. 2. The calculated flow rate data are in solid circles and their units are in mass flow rate per unit circumference of the pipe, in order to compare with the experiment. The corresponding high water flow case is presented in Fig. 6 . Discussion on the above will be made in the next section.

The circumferential water film flow rates for both the higher water flow case and the low water flow case are displayed in Fig. 7 , where there is no comparison with experiment. It shows a hunting pattern from about $1 \times 10^{-4} \mathrm{~kg} / \mathrm{s}$ at the pipe top to about $0.01 \mathrm{~kg} / \mathrm{s}$ at the bottom. The trend of the prediction is perhaps a ten-fold increase from $1 \times 10^{-4}$ to $1 \times 10^{-3} \mathrm{~kg} / \mathrm{s}$. The pattern may be due to the effect of taking finite sectors of the pipe in our calculations. An increase in the number of lateral mesh should smoothen out the points in Fig. 7 . However the computer time required for the studies will increase beyond the present CPU time of

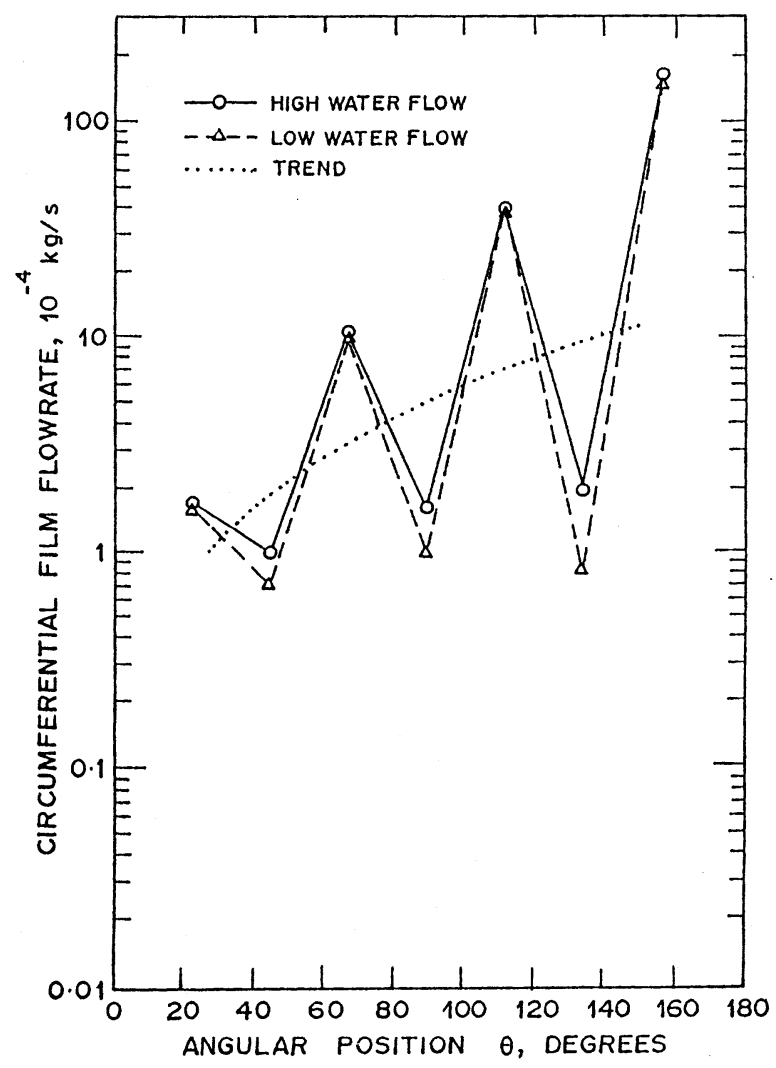

Fig. 7 Angular distribution for circumferential flow rates. 
30 hours (on the FACOM computer).

\section{DISCUSSION AND COMPARISON WITH EXPERIMENT}

There are three experimental lines in Fig. 5. The two experimental lines indicated by triangles are results of direct experimental measurements made with two different fin interception arrangements, and detailed in Butterworth and Pulling. Basically part of the axial flow is taken off through a porous window on the pipe within a pair of fins raised to a height of $7 \mathrm{~mm}$ or $3.5 \mathrm{~mm}$ (termed high fin and low fin respectively). The device is rotatable so that measurements can be made over the whole circumference. Also shown in Fig. 5 are the square points which are flow rates calculated by Butterworth and Pulling (1973) based on film thicknesses measured by the needle contact technique, and with the assistance of theoretical curves reported by Hewitt (1961). These curves were results of extending the analysis of Dukler (1959), who made one of the many models in the literature for the interrelationship between film thickness, film flow-rate and pressure gradient (Hewitt and Taylor, 1970). This third set gives the local values, whereas the first two sets give the average values over the width of the fins used for water extraction. It is seen that the present theoretical results (solid circles) are closer to this third line than those of direct measurements. Perhaps the needle contact technique for thickness is more reliable than the fin technique for flow rates, since the larger dimension fins may interfere more with the local flow field. Furthermore, the thickness measurements are predicted fairly well by the Code, as reported in the earlier paper. Nevertheless from $\theta=0^{\circ}$ to about $\theta=90^{\circ}$ the theoretical values are higher than the experimental values, and at the pipe bottom they are somewhat lower than experimental values.

In the case of low water flow in Fig. 6 , the predicted results seem to agree with the fin results, although they are all above the results calculated from thicknesses. If confidence is greater for the results calculated from thicknesses, based on the earlier arguments, then it is concluded that the prediction is higher than experimental

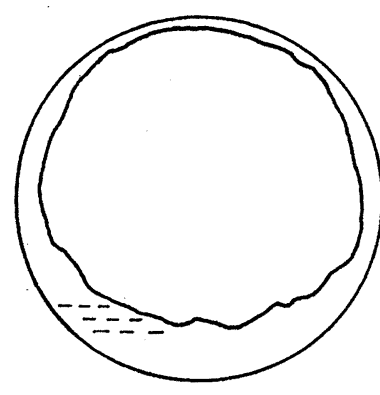

ACTUAL SECTION

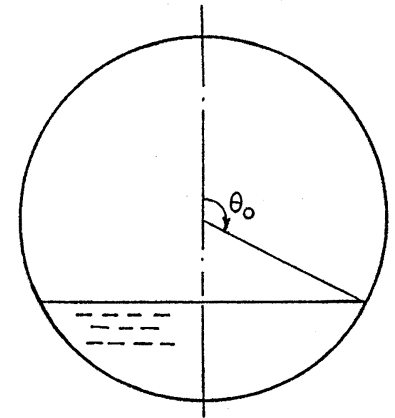

IDEALIZED SECTION

Fig. 8 Idealisation fo flow section. 
results. The rest of this section explains an attempt to correct the predicted results.

In the present analysis, the subchannel cross-section is of sectorial geometry extending from the wall to the pipe center. Information exchange in the local radial direction is not modelled by the code, with the result that there is no representation for axial velocity distribution perpendicular to the pipe wall and only a single axial velocity is represented. This may be particularly inadequate for the thin water film, where the wall effect is expected to reduce the water film velocity from the turbulent layer to the laminar sub-layer. Hence the Code is expected to over-predict the liquid water flow rate. The strategy adopted is to make appropriate corrections to the water film axial velocity in each subchannel taking into account the wall effect, and ensuring the conservation of water flow rate within the pipe. Figure 8 shows how the entire water film in the pipe is idealized by occupying only the bottom part of the pipe, and only those subchannels, deemed to have thin film and within the angular position $\theta_{0}$, will be corrected. The value of $\theta_{0}$ may be obtained from

$$
\theta_{o}-\left(1-\bar{\alpha}_{F}\right)-\frac{1}{2} \sin 2 \theta_{o}=0
$$

where $\bar{\alpha}_{\mathrm{F}}$ is the average water film volume fraction in the pipe.

Next, it is assumed that the actual water velocity profile in the pipe is the same as that for single-phase flow under turbulent diffusivity effect, and at steady, fully developed conditions. The usual three-layer velocity profile in a smooth pipe is thus taken as (von Kármán, 1939),

$$
\left.u_{F}^{+}\right|_{\text {actual }}=\left\{\begin{array}{lll}
y^{+} & , y^{+} \leqq 5 \\
5 \ln y^{+}-3.05 & , \quad 5<y^{+} \leqq 30 \quad \ldots \ldots \ldots \ldots \ldots \ldots \ldots \ldots \ldots \ldots \ldots \ldots \\
2.5 \ln y^{+}+5.5 & , y^{+}>30
\end{array}\right.
$$

where $u_{F}{ }^{+}=$dimensionless axial film velocity, $u_{F} /\left(\tau_{o} / \rho_{F}\right)^{1 / 2}$

$y^{+}=$dimensionless distance from wall, $y\left(\tau_{o} / \rho_{F}\right)^{1 / 2} / \nu_{F}$

$\tau_{0}=$ wall shear stress

$\rho_{F}=$ density of water

$\nu_{F}=$ kinematic viscosity of water

$u_{F}=$ axial velocity of water film

$y=$ perpendicular distance from wall.

This velocity profile may be integrated over the film thickness $\delta$ to give the mass flow rate, and hence an average velocity may be defined as

$$
\left.\bar{u}_{F}^{+}\right|_{\text {actual }}=\frac{1}{\delta^{+}} \int_{0}^{\delta^{+}} u^{+} d y^{+}
$$

where ${\overline{u_{F}}}^{+}=$average dimensionless velocity

$\delta^{+}=$dimensionless film thickness, $\delta\left(\tau_{o} / \rho_{F}\right)^{1 / 2} / \nu_{F}$. 
Carrying out the integration yields

$$
\left.\bar{u}_{F}^{+}\right|_{\text {actual }}= \begin{cases}\frac{1}{2} \delta^{+} & , \quad \delta^{+} \leqq 5 \\ 5 \ln \delta^{+}+\frac{12.5}{\delta^{+}}-8.05 & , \quad 5<\delta^{+} \leqq 30 \\ 2.5 \ln \delta^{+}-\frac{64}{\delta^{+}}+3 & \ldots .\end{cases}
$$

It is now assumed that the code-calculated velocity has a single value as that at the pipe center. Although the water film does not really extend to the center of the pipe, the code does not make this differentiation, and hence the justification of this assumption. Thus

$$
\left.{\overline{u_{F}}}^{+}\right|_{\text {calculated }}=2.5 \ln (1458)+5.5=23.71
$$

The first correction factor $C_{1}$ to be multiplied to the code results is then defined as

$$
C_{1}=\bar{u}_{F}+\left.\right|_{\text {actual }} \div\left.{\overline{u_{F}}}^{+}\right|_{\text {calculated }}
$$

In order to compensate for the decrease in flowrates caused by the first correction, a second multiplication correction factor $C_{2}$ is needed.

This is defined by

$$
\begin{array}{cc}
C_{2} \times \text { [sum of first-corrected } & =\text { [sum of uncorrected } \\
\text { flow rates in subchannels }] & \text { flow rates in subchannels }] \cdots
\end{array}
$$

Symbolically,

$$
C_{2}\left[\sum \delta \times\left.{\overline{u_{F}}}^{+}\right|_{\text {actual }}\right] \div\left[\sum \delta \times C_{1} \times\left.{\overline{u_{F}}}^{+}\right|_{\text {actual }}\right]
$$

where the summation is over all subchannels.

The above procedure is implemented by a subroutine program which is called after the termination of calculation by the FIDAS Code. In the program, the node pertaining to the idealization in Fig. 8 is first determined, followed by the calculation on film thicknesses. Then the velocity profiles are put in and the first correction factors are determined. This is followed by the determination of the second correction factor and the calculation of water flow rates.

The results of the above augmentation are shown in Fig. 5 and Fig. 6 as open circles. It is seen that the corrected prediction now lie much closer to the experimental results based on thickness measurements. 


\section{CONCLUDING REMARK}

It is concluded that the application of the conservation equations to control volumes in subchannels is one of the appropriate ways to analyse horizontal annular flow. In this approach the solutions for the formulated velocities take care of circumferential or recirculating flow in the pipe. The combined effect of the entrainment and deposition, as well as diffusion and interfacial forces play the role in causing the existence of the upper film, in this model. A corrective procedure has been taken to compensate for the lack of radial velocity variation in the model. This is obviously easier to implement than to re-write the Code to include a full three-component velocity model. It is noteworthy that the errors in the experimetal flow rates are not known. Similarly the accuracy of the analysis is affected not only by the modelling, but also by constitutive relations. Nevertheless the assumption of simple constitutive relations adopted here appears to work rather well on the whole, in view of the limitations of the model.

\section{ACKNOWLEDGEMENT}

The assistance given by $\mathrm{K}$. Watanabe in the present work is gratefully acknowledged. The contribution by one of us (C. P. T.) was made possible through an award by the Japan Science and Technology Agency.

\section{REFERENCES}

Bennett, A.W., Hewitt, G.F., Kearsey, H.A., Keeys, R.K.F. and Pulling, D.J. (1966) : Studies of Burnout in Boiling Heat Transfer to Water in Round Tubes with Non-uniform Heating. UKAEA Report No. AERE-R5076, U.K. .

Butterworth, D. (1972): Air-water Annular Flow in a Horizontal Tube. Prog. Heat Mass Transfer 6, 235-251.

Betterworth, D. (1973) : An Analysis of Film Flow for Horizontal Annular Flow and Condensation in a Horizontal Tube. UKAEA Report No. AERE-R7575, U.K. .

Butterworth, D. and Pulling, D.J. (1973) : Film Flow and Film Thickness Measurements for Horizontal, Annular, Air-water flow. UKAEA Report No. AERE-R7576, U.K. . Chaddock, J.B. (1957) : Film Condensation of Vapour in a Horizontal Tube. Refrigerating Engng., 36-41, 90-95, April 1957.

Chato, J.C. (1962) : Laminar Condensation inside Horizontal and Inclined Tubes. ASHRAE Journal. 52-60, Feb. 1962.

Dukler, A.E. (1959) : Fluid Mechanics and Heat Transfer in Falling Film Systems. Paper presented at the ASME-AICHE 3rd National Heat Transfer Conference, August 1959. See also Chem. Eng. Prog. Symp. Series 56, 30, 1-10(1960).

Farmer, R., Griffith, P. and Rohsenow, W.M. (1970) : Liquid Droplet Deposition in Two-phase Flow. Trans. ASME, Ser. C, 92-4, 587-594

FIDAS-3DT Code 1987. Internal Report, Power Reactor and Nuclear Fuel Corporation, O-arai Engineering Center, O-arai, Japan (in Japanese). 
Fukano, T. and Ousaka, A. (1989) : Prediction of the Circumferential Distribution of Film Thickness in Horizontal and Near-horizontal Gas-liquid Annular Flows, Int. J. Multiphase Flow 15, 403-419.

Hewitt, G.F. (1971) : Analysis of Annular Two-phase Flow : Application of the Dukler Analysis to Vertical Flow in a Tube. UKAEA Report No. AERE-R3680.

Hewitt, G.F. and Hall-Taylor, N.S. (1970): Annular Two-Phase Flow. Pergamon Press, Oxford.

Hutchinson, P., Butterworth, D. and Owen, R.G. (1974) : Development of a Model for Horizontal Annular Flow. UKAEA Report No. AERE-R7789, U.K. .

Hutchinson, P. and Whalley, P.B. (1972) : A Possible Characterization of Entrainment in Annular Flow. UKAEA Report No. AERE-R7126, U.K..

James, P.W. and Burns, A. (1979) : Further Developments in the Modelling of Horizontal Annular Flow. UKAEA Report No. AERE-R9373, U.K. .

James, P.W., Wilkes, N.S., Conkie, W. and Burns, A. (1987): Developments in the Modelling of Horizontal Annular Two-phase Flow. Int. J. Multiphase Flow 13, 173-198.

Kataoka, I. and Ishii, M. (1982) : Mechanism and Correlation of Droplet Entrainment and Deposition in Annular Two-phase Flow. Report ANL-82-44, U.S.A. .

Laurinat, J.E. (1978) : Studies of the Effects of Pipe Size on Horizontal Annular Two-phase Flows. Ph.D. Thesis, University of Illinois, Urbana, U.S.A..

Lin, T.F., Jones Jr., O.C., Lahey Jr., R.T., Block, R.C. and Murase, M. (1985) : Film Thickness Measurements and Modelling in Horizontal Annular Flows. Physico-Chemical Hydrodynamics 6, No.1/2, 197-206.

Morooka, S., Ishizuka, T. and Kagawa, T. (1984) : Development of POST-MULTI Code for the Analysis of Heat Transfer beyond Boiling Transition. J. Atomic Energy Soc. Japan 26, 136-138(in Japanese).

Paleev, I.I. and Filippovich, B.S. (1966): Phenomena of Liquid Transfer in Two-phase Dispersed Annular Flow. Int. J. Heat Mass Transfer 9, 1089-1093.

Saito, T., Huges, E.D. and Carbon, M.W. (1978) : Multi-fluid Modelling of Annular Two-phase Flow. Nucl. Engng Des. 50, 225-271.

Sugawara, S. (1988) : Droplet Deposition and Entrainment Modelling Based on the Three-Fluid Model. In Proc. 3rd Int. Top. Meet. on Nuclear Power Plant Thermal Hydraulics and Operations, Seoul, Korea, Vol.A1, 19-28.

Sugawara, S. and Miyamoto, Y. (1990): FIDAS : Detailed Subchannel Analysis Code Based on the Three-fluid and Three-field Model, Nucl. Eng. and Design 120, 147-161. Sugawara, S., Sakai, T., Watanabe, K. and Rummens, H.E.C. (1989): Subchannel Analysis by the FIDAS Code Based on the Three-fluid Model, Proc. 4th Int. Topical Meeting on Nuclear Reactor ThermalHydraulics Vol.1, Karlsruhe, F.R.G., Oct. 10-13, 1989, 560-567.

Scott, D.S. (1963) : Properties of Cocurrent Gas-liquid Flow. Chem. Engng. 4, 199-277. Tso, C.P. and Sugawara, S. (1989): An Analysis of Annular Two-phase Three-field Horizontal Flow, Transport Phenomena in Thermal Control, Hemisphere Publishing 
Corp., New York, 701-708.

Tso, C.P. and Sugawara, S. (1990) : Film Thickness Prediction in a Horizontal Annualr Two-phase Flow, Int. J. Multiphase Flow 16, 867-884.

Ueda, T. (1981): Two-Phase Flow-Flow and Hear Transfer. Yokendo, Japan (in Japanese).

von Kármán, T. (1939) : The Analog between Fluid Friction and Heat Transfer, Trans. ASME 61, 705-710.

Wallis, G.B. (1969) : One-dimensional Two-phase Flow. McGraw-Hill, New York.

Whalley, P.B. (1977): The Calculation of Dryout in Rod Bundles, Int. J. Multiphase Flow 3, 501-515.

Wilkes, N.S., Conkie, W. and James, P.W. (1980) : A Model for the Droplet Deposition Rate in Horizontal Annular Two-phase Flow. UKAEA Report No. AERE-R9691, U.K.. 


\section{Appendix A}

\section{CONSERVATIVE EQUATIONS}

The following equations are applied to the water film in the unit cells. They are written in the general form to include the effect of heat transfer amongst the fluids, as these general forms are actually used in the Code for this adiabatic problem. Subscripts $F, E$ and $G$ refer to the water-film, entrained water droplets, and air respectively.

\section{Mass Conservation Equation}

$$
\begin{aligned}
& \frac{\partial}{\partial t}\left(\alpha_{F} \rho_{F}\right)=-\frac{1}{A} \frac{\partial}{\partial x}\left(\alpha_{F} \rho_{F} u_{F} A\right)+\frac{1}{A} \sum_{k}\left(\alpha_{F} \rho_{F} v_{F} S_{L}\right)_{k} \\
& -\left(\Gamma_{F G}-\Gamma_{G F}+\dddot{m}_{F G}\right)+\frac{S}{A}\left(m_{D}-m_{E}\right) \\
& +\frac{\partial}{\partial x}\left[\alpha_{F}\left(D_{C F}+D_{C F}^{T}\right) \frac{\partial \rho_{F}}{\partial x}\right]-\frac{1}{A} \sum_{k}\left[S_{L} \alpha_{F}\left(D_{C F}+D_{C F}^{T}\right) \frac{\partial \rho_{F}}{\partial L}\right]_{k}
\end{aligned}
$$

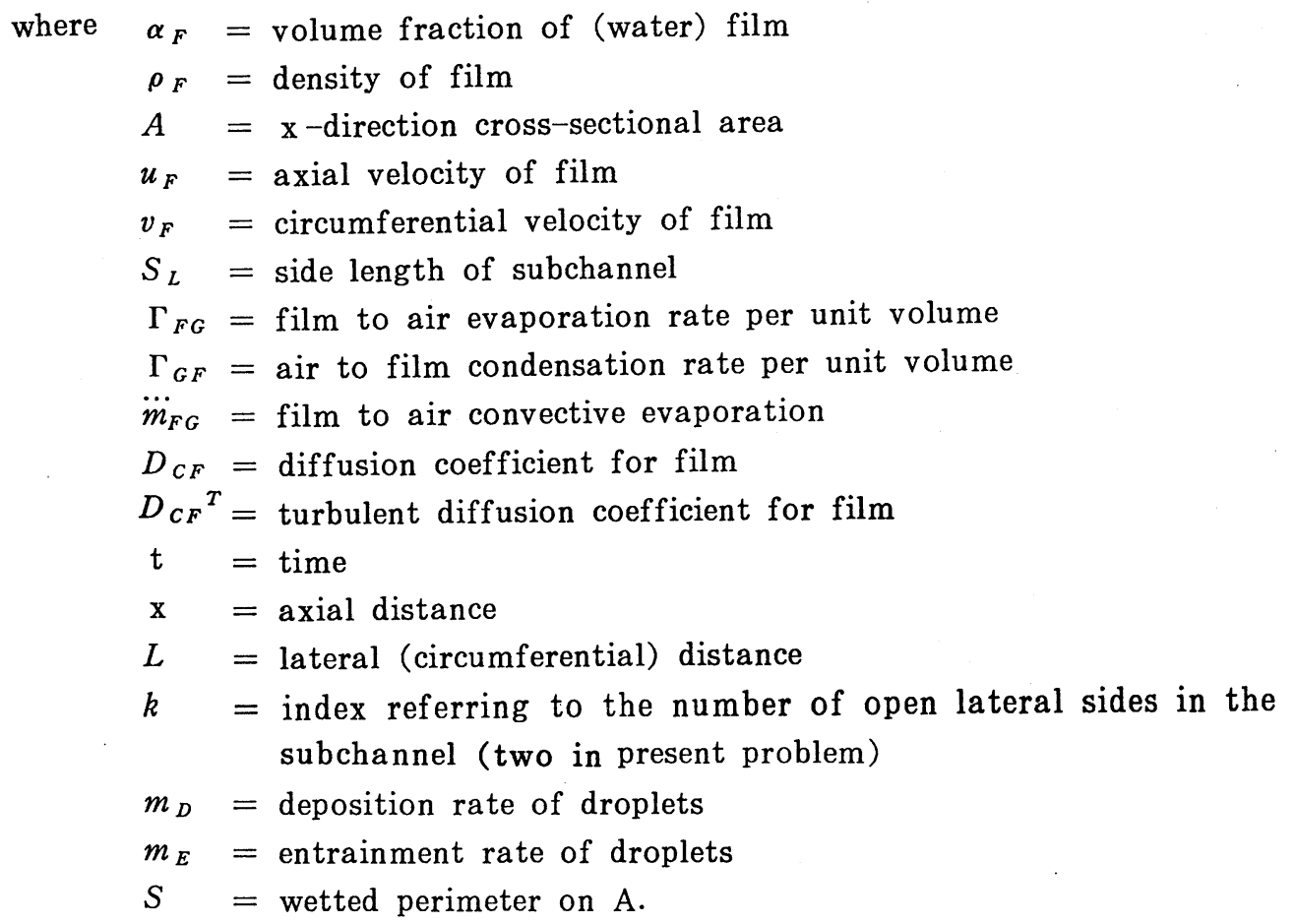




\section{Momentum Conservation Equations}

There are two equations for each fluid, in the $\mathbf{x}$-and $\mathrm{L}$-directions and referred to by subscripts $x$ and $L$ respectively. For the water film, the $\mathrm{x}$-direction equation is

$$
\begin{aligned}
\frac{\partial}{\partial t}\left(\alpha_{F} \rho_{F} u_{F}\right)= & -\frac{\partial}{\partial x}\left(\alpha_{F} \rho_{F} u_{F}^{2}\right)+\frac{1}{A} \sum_{k}\left(\alpha_{F} \rho_{F} v_{F} u_{F} S_{L}\right)_{k} \\
& -\alpha_{F} \frac{\partial P}{\partial x}-\alpha_{F} \rho_{F} g_{x}-\dddot{\tau}_{W F x}+\dddot{\tau}_{F G x}-\dddot{\tau}_{E F x} \\
& -\left(\Gamma_{F G}+\dddot{m}_{F G}\right) u_{F}+\Gamma_{G F} u_{G}+\frac{S}{A}\left(m_{D} u_{E}-m_{E} u_{F}\right) \\
& -\frac{\partial}{\partial x}\left(\alpha_{G} \tau_{F x x}\right)+\frac{1}{A} \sum_{k}\left(S_{L} \alpha_{E} \tau_{F L x}\right)_{k} \cdots \cdots
\end{aligned}
$$

where $P=$ Pressure

$\dddot{\tau}_{W F x}=$ wall to film shear force per unit volume

$\dddot{\tau}_{F G x}=$ film to air shear force per unit volume

$\dddot{\tau}_{E F x}=$ droplets to film shear force per unit volume

$\tau_{F x x}=$ axial contribution of axial molecular and turbulent viscous stress

$\tau_{F L x}=$ axial contribution of lateral molecular and turbulent viscous stress

$g_{x}=$ gravitational acceleration(in the $\mathrm{x}$-direction).

Similarly, the $L$-direction equation is

$$
\begin{aligned}
\frac{\partial}{\partial t}\left(\alpha_{F} \rho_{F} v_{F}\right)= & -\frac{\partial}{\partial x}\left(\alpha_{F} \rho_{F} v_{F} u_{F}\right)-\frac{\partial}{\partial L}\left(\alpha_{F} \rho_{F} v_{F}^{2}\right) \\
& -\alpha_{F} \frac{\partial P}{\partial L}-\alpha_{F} \rho_{F} g_{L}-\dddot{\tau}_{W F L}+\dddot{\tau}_{F G L}-\dddot{\tau}_{E F L} \\
& -\left(\Gamma_{F G}+\dddot{m}_{F G}\right) v_{F}+\Gamma_{G F} v_{G}+\frac{S}{A}\left(m_{D} v_{E}-m_{E} v_{E}\right) \\
& -\frac{\partial}{\partial L}\left(\alpha_{F} \tau_{F L L}\right)+\frac{\partial}{\partial x}\left(\alpha_{F} \tau_{F L x}\right)
\end{aligned}
$$

where $\tau_{F L L}=$ lateral contribution of lateral molecular and turbulent viscous stress.

\section{Energy Equation}

This is given by 


$$
\begin{aligned}
\frac{\partial}{\partial t}\left(\alpha_{F} \rho_{F} h_{F}\right)= & -\frac{1}{A} \frac{\partial}{\partial x}\left(\alpha_{F} \rho_{F} u_{F} A h_{F}\right)+\frac{1}{A} \sum_{\mathrm{k}}\left(\alpha_{F} \rho_{F} v_{F} S_{L} h_{F}\right)_{\mathrm{k}} \\
& +\left(\Gamma_{G F} h_{l s}-\Gamma_{F G} h_{g s}-\dddot{m}_{F G} h_{g s}\right)+\frac{S}{A}\left(m_{D} h_{E}-m_{E} h_{F}\right) \\
& +\dddot{q}_{I F}-\frac{\partial}{\partial x}\left(\alpha_{F} q_{F x}\right)+\frac{1}{A} \sum_{\mathbf{k}}\left(S_{L} \alpha_{F} q_{F L}\right)_{\mathrm{k}}+\dddot{Q}_{W F}
\end{aligned}
$$

where $\quad h_{G}=$ specific enthalpy of film

$h_{l s}=$ liquid-saturated specific enthalpy

$h_{g s}=$ gas-saturated specific enthalpy

$\dddot{q}_{I F}=$ heat transfer rate to film from adjacent open sides

$q_{F x}=$ thermal diffusion rate to film in the $x$-direction

$q_{F L}=$ thermal diffusion rate to film in the $L$-direction

$\dddot{Q}_{W F}=$ heat transfer rate to film from the wall.

Similar equations as ( A 1 ) to (A 4 ) may be written for the entrained water droplets and the air. 


\section{Appendix B}

\section{CONSTITUTIVE EQUATIONS}

\section{(a) Droplet entrainment rate}

Ueda's model (1981) is used. This is based on Ueda's experimental air-water and air-alcohol results, where the entrainment rate in $\mathrm{kg} / \mathrm{m}^{2}$ is

$$
m_{E}=3.54 \times 10^{-3} U^{0.57} \text {, when } U>120 \text {, }
$$

where

$$
\begin{array}{ll}
U & =\frac{\left|\tau_{F G}\right|}{\sigma}\left(\frac{\alpha_{F} u_{F}}{\sigma}\right) 0.6 \\
\sigma & =\text { air-water surface tension, in } \mathrm{N} / \mathrm{m} \\
\tau_{F G} & =\text { air-water shear stress, in } \mathrm{N} / \mathrm{m}^{2}
\end{array}
$$

(b) Droplet deposition rate

Using the model by Farmer et al. (1970), the deposition is given by

$$
m_{D}=k_{f} C \text {, }
$$

where $k_{f}$ and $C$ are the droplet transfer coefficient and average droplet density given by

$$
\begin{aligned}
& k_{f}=(1 / 4) \lambda D u_{G}, \text { in } \mathrm{m} / \mathrm{s}, \\
& C=\alpha_{E} \rho_{E} /\left(\alpha_{E}+\alpha_{G}\right), \text { in } \mathrm{kg} / \mathrm{m}^{3},
\end{aligned}
$$

where $\lambda=$ a constant dependent on droplet diameter $\delta_{D}$, in $1 / \mathrm{m}$

$$
D=\text { pipe inner diameter, in } \mathrm{m} \text {. }
$$

\section{(c) Wall-liquid drag force}

This is based on Blasius' form as illustrated by the follwing one-dimensional case :

$$
\begin{aligned}
\dddot{\tau}_{W F}= & \bar{A}_{W F} K_{W F} \frac{\rho_{F}}{2} u_{F}\left|u_{F}\right| \text {, in } \mathrm{Pa} / \mathrm{m}, \\
\bar{A}_{W F} & =\text { wall-liquid average area } \\
& =4 / \mathrm{D}, \text { in } \mathrm{l} / \mathrm{m} \\
K_{W F} & =\text { Blasius' expression } \\
& =\frac{1}{4}\left(\frac{0.316}{\operatorname{Re}_{F}^{0.25}}\right), \text { dimensionless, }
\end{aligned}
$$

where $R e_{F}=$ liquid film Reynolds number

$$
=u_{F} D / \nu_{F}
$$


$\nu_{F} \quad=$ kinetic viscosity of liquid film.

(d) Vapor-liquid drag force

This uses Ueda's model (1981) as shown by the following one-dimensional case :

$$
\dddot{\tau}_{F G}=\bar{A}_{F G} K_{F G} \frac{\rho_{G}}{2}\left(u_{G}-u_{F}\right)\left|u_{G}-u_{F}\right| \text {, }
$$

where $\quad \bar{A}_{F G}=$ vapor-liquid interfacial average area

$$
=(4 / D)\left(1-\alpha_{F}\right)^{1 / 2} \text {, in } 1 / \mathrm{m}
$$

$$
\begin{aligned}
K_{F G}= & 1 / 4 \times\left(2.85-2.10 \alpha_{G}{ }^{2.20}\right)^{4}\left[\frac{\left(\rho_{F}-\rho_{G}\right) g D_{F G}}{\rho_{G}\left(u_{G}-u_{F}\right)^{2}}\right]^{0.7} \\
& \times\left[\frac{\mu_{G}\left|u_{G}-u_{F}\right|}{\mu_{F} u_{F}}\right]^{0.1}\left[\frac{\left|u_{G}-u_{F}\right| D_{F G}}{\nu_{G}}\right]^{-0.2} \times C_{H}
\end{aligned}
$$

$D_{F G}=$ diameter of vapor-droplet flow section

$C_{H}=$ a correction factor for horizontal flow taken to be 2

$\mu, \nu=$ dynamic and kinematic viscosity, respectively .

\section{(e) Vapor-droplet drag force}

Again, in the one-dimensional case, the vapor-droplet drag force is

$$
\dddot{\tau}_{E G}=\bar{A}_{E G} K_{E G} \frac{\rho_{G}}{2}\left(u_{G}-u_{E}\right)\left|u_{G}-u_{E}\right|,
$$

where $\bar{A}_{E G}=$ vapor-droplet area (total cross-sectional droplet area)

$$
\begin{aligned}
& =3 \alpha_{E} /\left(2 \delta_{D}\right) \\
\delta_{D} & =\text { droplet diameter } \\
K_{E G} & =\frac{24}{R e_{\delta}}\left(1+0.15 R e_{\delta}{ }^{0.687}\right)+\frac{0.42}{1+4.25 \times 10^{4} R e_{\delta}{ }^{-1.16}}
\end{aligned}
$$

(Morooka et al. 1984)

$$
R e_{\delta}=\left|u_{G}-u_{E}\right| \delta_{D} / \nu_{E} \text {. }
$$

(f) Droplet size

The droplet size is given by

$$
\delta_{D}=\left\{\begin{array}{l}
\frac{\sigma \mathrm{We}}{\rho_{\mathrm{G}}\left(\mathrm{u}_{\mathrm{G}}-\mathrm{u}_{\mathrm{E}}\right)^{2}} \\
10^{-3}, \text { if } \delta_{D}<10^{-3}
\end{array}\right.
$$

where $W e=$ Weber number. 
(g) Molecular and turbulent viscous stress

The following appear in equations (A 2) and (A 3) :

$$
\begin{aligned}
& \tau_{F x x}=-2\left(\mu_{F}+\mu_{F}^{T}\right) \frac{\partial u_{F}}{\partial x}+3 \rho_{F} l_{m}^{2} \sum_{\mathbf{k}}\left(\frac{\partial u_{F}}{\partial L}+\frac{\partial v_{F}}{\partial x}\right)_{\mathbf{k}}{ }^{2} \\
& \tau_{F L x}=-\left(\mu_{F}+\mu_{F}^{T}\right)\left(\frac{\partial u_{F}}{\partial L}+\frac{\partial v_{F}}{\partial x}\right) \\
& \tau_{F L L}=-2\left(\mu_{F}+\mu_{F}^{T}\right) \frac{\partial v_{F}}{\partial L}+3 \rho_{F} l_{m}{ }^{2} \sum_{\mathbf{k}}\left(\frac{\partial u_{F}}{\partial L}+\frac{\partial v_{F}}{\partial x}\right)_{\mathbf{k}}{ }^{2},
\end{aligned}
$$

where $\mu_{F}=$ dynamic viscosity of film

$\mu_{F}{ }^{T}=$ turbulent dynamic viscosity of film

$$
=\rho_{F} l_{m}^{2}\left[\sum_{\mathbf{k}}\left(\frac{\partial u_{F}}{\partial L}+\frac{\partial v_{F}}{\partial x}\right)_{\mathbf{k}}^{2}\right]^{1 / 2}
$$

$l_{m}=$ momentum mixing length, taken as the equivalent diameter. 